\title{
Reference Ellipsoid Parameters of Cameroon from GPS Data
}

\author{
Ludovic Houetchak Kande*, Joseph Kamguia, Loudi Yap, Louis Foyang \\ National Institute of Cartography, Yaounde, Cameroon \\ Email: "Ikande2001@yahoo.fr
}

Received 13 July 2016; accepted 16 August 2016; published 19 August 2016

Copyright (C) 2016 by authors and Scientific Research Publishing Inc.

This work is licensed under the Creative Commons Attribution International License (CC BY). http://creativecommons.org/licenses/by/4.0/

(c) () Open Access

\section{Abstract}

The standard spheroidal reference surface also referred as the reference ellipsoid is a part of mapping basic infrastructures of a country. In Cameroon, the ellipsoid Clarke 1880 with unknown parameters used for a long time as reference system in basic mapping, has lead to the use of World Geodetic System 1984 (WGS 84) whose parameters are well known. Meanwhile, the latter is not accurate locally due to the fact that the best ellipsoid is the one that fits very well with the local geoid. In order to look for the parameters of the local ellipsoid that fits best to the local geoid (Cameroon Geoid Model 2011 (CGM11)), the Global Positionning System (GPS) data made of 525 geodetic ground control points of the new geodetic network of Cameroon set up in 2011 were used. These GPS measures provide for each point the values of the ellipsoid height and the elevation which are used to determine the parameters of the local ellipsoid model for Cameroon through the least square form of the Molodensky analytical method. The results are given as the difference in ellipsoidal height relative to the parameters of the WGS84 ellipsoid assuming the two ellipsoids in their parallel. These results show that the obtained ellipsoid fits better to the local geoid with $1.072 \mathbf{m}$ as the standard deviation value, is improved considerably in comparison to the previous studies conducted in Cameroon whose standard deviation was fairly equal to $1.679 \mathrm{~m}$.

\section{Keywords}

Ellipsoid, Parameters, Geoid, GPS, Molodensky Analytical Method, Cameroon

\section{Introduction}

The basis cartographic infrastructure is characterized by a mapping reference system made of the ellipsoid, representing the mathematical model of the earth surface. This model is determined by a set of parameters that

\footnotetext{
*Corresponding author.
} 
define its shape, position and orientation in space. The reference system is determined by selecting a set of points called triangulation points in the area of interested. These points determined by the coordinate observations constitute the frame of the map. It's from these coordinates that the projection system is developed, on which all the features to be mapped are located. In Cameroon, the works of IGN France effectively started in 1939 with the Gardet triangulation on the Cameroon-Nigeria border and ended in 1976, enabled the production of 44 map cuts at scale 1:200,000 and 248 map cuts at scale 1:50,000. Since these first geodetic works, based on the obsolete basic infrastructures and several lines of leveling crossing the major roads at that period, multiple mapping systems have emerged. In fact, due to the lack of reliable cartographic infrastructure, we can say that each mapping project has raised its reference system. Most of these reference systems use the ellipsoid Clarke 1880 whose origin is poorly known, and its structure is made of astronomical points with no connection between them [1]. On the other hand, the newer systems use the WGS84 ellipsoid as reference in combination with new geodetic network of Cameroon. The WGS 84 reference ellipsoid model is calculated to represent the entire earth surface. It reflects the general shapes of the earth but does not take into account all the local topographic characteristics of each country like the case of Cameroon [2]. So far, the best ellipsoid model for a country is the one that fits very well to the local geoid [3]. The Molodensky simplified formula enables the transformation of the coordinates of a point between two different ellipsoid parameters when processing parameters are known [4]-[6]. In this case we are looking for a local ellipsoid parameters in Cameroon knowing the parameters of a global WGS84 ellipsoid and with the GPS data made of 525 points of the geodetic network of Cameroon. This problem is solved by applying the least squares method to the simplified formula Molodensky [7].

\section{Method}

Consider an oriented ellipsoid, centered to $(X, Y, Z)$ with the parameters $(a, f)$. Let also a point $\mathrm{P}$ with the coordinates $(\varphi, \lambda, h)$. The ellipsoidal height difference $\delta h$ relative to a second ellipsoid, centered to $(X+\Delta X, Y+\Delta Y$, $Z+\Delta Z)$ with the same orientation and the parameters $\left(a^{\prime}, f^{\prime}\right)$ is given. The Molodensky formula helps to establish a relationship between the coordinates of the point $P$ in both ellipsoids [5] [8]. Generally, the simplified form of this formula is used for the ellipsoidal height difference which is given by the relation:

$$
\Delta h=\cos \varphi \cos \lambda \Delta x+\cos \varphi \sin \lambda \Delta y+\sin \varphi \Delta z+(a \Delta f+f \Delta a) \sin ^{2} \varphi-\Delta a
$$

$$
h=\text { ellipsoid height (meters) }
$$

$\varphi=$ latitude

$\lambda=$ longitude

$a=$ semi-major axis of the spheroid (meters)

$b=$ semi-minor axis of the spheroid (meters)

$f=$ flattening of the spheroid.

In this study, we assume that the parameters of the first ellipsoid are well known and we look for the parameters of the second ellipsoid that fits best to the local geoid. For this, we measure a set of GPS points of the geodetic network evenly divided over the country. For each point Pi, the coordinates $\left(\varphi_{i}, \lambda_{i}, h_{i}\right)$ and its elevation $H_{0 i}$ (the height from the geoid) are known. It is assumed that the gap between the ellipsoid whose parameters are sought and the local geoid is the lowest possible. Using the least squares method, the relationship (1) becomes:

$$
\begin{aligned}
& \sum_{i=1}^{n}\left[\left(\cos \varphi_{i} \cos \lambda_{i} \Delta x+\cos \varphi_{i} \sin \lambda_{i} \Delta y+\sin \varphi_{i} \Delta z+a \sin ^{2} \varphi_{i}\right.\right. \\
& \left.\left.+a f \sin ^{2} \varphi_{i} \Delta f-\left(f \sin ^{2} \varphi_{i}-1\right) \Delta a\right)-\left(h_{i}-H_{0 i}\right)\right]=\min
\end{aligned}
$$

Equation (2) is in the form:

$$
\sum_{i=1}^{n}\left[f\left(\varphi_{i}, \lambda_{i}\right)-\Delta h_{i}\right]=\min
$$

With

$$
\begin{gathered}
f\left(\varphi_{i}, \lambda_{i}\right)=\left(\cos \varphi_{i} \cos \lambda_{i} \Delta x+\cos \varphi_{i} \sin \lambda_{i} \Delta y+\sin \varphi_{i} \Delta z+a \sin ^{2} \varphi_{i}+a f \sin ^{2} \varphi_{i} \Delta f-\left(f \sin ^{2} \varphi_{i}-1\right) \Delta a\right) \\
\Delta h_{i}=h_{i}-H_{0 i}
\end{gathered}
$$

Applying the partial derivatives to the coefficients of this function we have: 


$$
\frac{\partial}{\partial q_{j}} \sum_{i=1}^{n}\left[f\left(\varphi_{i}, \lambda_{i}\right)-\Delta h_{i}\right]^{2}=0
$$

where

$$
j=[1,5] ; q_{1}=\Delta x ; q_{2}=\Delta y ; q_{3}=\Delta z ; q_{4}=\Delta f ; q_{5}=\Delta a
$$

Which provides an equation in matrix form $A Q=B$. $A$ is a $5 \times 5$ symmetric matrix, $Q$ the self-column matrix with 6 coefficients whose values are to be determined and $B$, a self-column matrix. The coefficient of $A$ and $B$ are the following:

$$
\begin{aligned}
& A_{11}=\sum_{i=1}^{n} \cos ^{2} \varphi_{i} \cos ^{2} \lambda_{i} \\
& A_{12}=\sum_{i=1}^{n} \cos ^{2} \varphi_{i} \cos \lambda_{i} \sin \lambda_{i} \\
& A_{13}=\sum_{i=1}^{n} \cos \varphi_{i} \sin \varphi_{i} \cos \lambda_{i} \\
& A_{14}=\sum_{i=1}^{n} a \cos \varphi_{i} \sin ^{2} \varphi_{i} \cos \lambda_{i} \\
& A_{15}=\sum_{i=1}^{n} \cos \varphi_{i} \cos \lambda_{i}\left(f \sin ^{2} \varphi_{i}-1\right) \\
& A_{25}=\sum_{i=1}^{n} \cos \varphi_{i} \sin \lambda_{i}\left(f \sin ^{2} \varphi_{i}-1\right) \\
& A_{33}=\sum_{i=1}^{n} \sin { }^{2} \varphi_{i} \\
& A_{34}=\sum_{i=1}^{n} a \sin ^{3} \varphi_{i} \\
& A_{35}=\sum_{i=1}^{n} \sin \varphi_{i}\left(f \sin ^{2} \varphi_{i}-1\right) \\
& A_{44}=\sum_{i=1}^{n} a^{2} \sin ^{4} \varphi_{i} \\
& A_{45}=\sum_{i=1}^{n} a \sin ^{2} \varphi_{i}\left(f \sin ^{2} \varphi_{i}-1\right) \\
& A_{55}=\sum_{i=1}^{n}\left(f \sin ^{2} \varphi_{i}-1\right)^{2} \\
& B_{1}=\sum_{i=1}^{n} \cos \varphi_{i} \cos \lambda_{i} \Delta h \\
& B_{2}=\sum_{i=1}^{n} \cos \varphi_{i} \sin \lambda_{i} \Delta h \\
& B_{3}=\sum_{i=1}^{n} \sin \varphi_{i} \Delta h \\
& B_{4}=\sum_{i=1}^{n} a \sin ^{2} \varphi_{i} \Delta h \\
& B_{5}=\sum_{i=1}^{n}\left(f \sin ^{2} \varphi_{i}-1\right) \Delta h \\
& A_{i=1}
\end{aligned}
$$

The resolution of this equation enables us to determine the values of $\Delta X, \Delta Y, \Delta Z, \Delta f$ and $\Delta a$. The $R_{i}=f\left(\varphi_{i}, \lambda_{i}\right)-\Delta h_{i}$ is the residue between model and measurement and also corresponds to the difference between the ellipsoid and the geoid assuming that each point of GPS measurement is reduced to the geoid level.

The uncertainties are not known for each measurement. The best estimate of the variance is given by the equation:

$$
\sigma_{0}^{2}=\frac{1}{N-M} \sum_{i=1}^{N} R_{i}^{2}
$$

$N-M$ is the number of degree of freedom model/measurement and $\sigma_{0}$ the standard deviation. 


\section{Data Processing, Results and Discussion}

The data used in this study are the GPS coordinates of the geodetic network of Cameroon established in 2011 [9]. Its structure consists of 25 reference points, 510 base points and 20 auxiliary points observed from GPS technology. All these points (Figure 1) are determined on the WGS 84 reference system where origin is in the center of the earth mass with its parameters indicated in the Table 1. It is important to note that the reference points constitute the primary geodetic network (first order network) and are situated at about $200 \mathrm{~km}$ each other. The base points make up the second order network are composed of spaced points of 20 to $30 \mathrm{~km}$ and the auxiliary points enable the joining all the network.

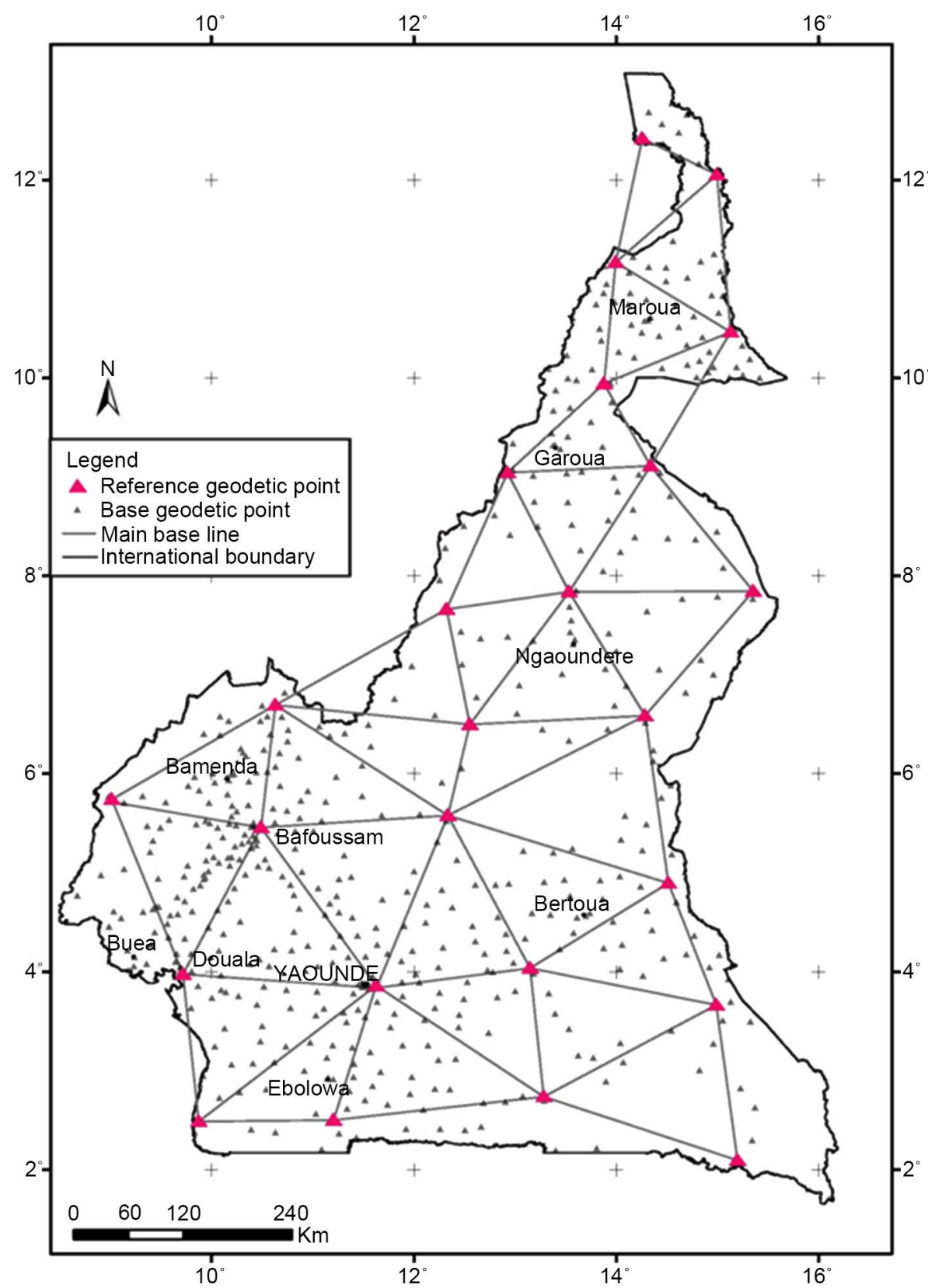

Figure 1. Structure of the New Geodetic Network Cameroon elaborated by [7]. The small pink triangles show the reference geodetic points and the small black triangles represent the base geodetic points. Data are densely distributed in the west, but there are many gaps in the central and eastern parts of the country. 
Table 1. Parameters of the WGS84 ellipsoid.

\begin{tabular}{ccc}
\hline Parameter & WGS84 & GRS80 \\
\hline Semi-major axis of the spheroid $(a)$ & $6,378,137.0000 \mathrm{~m}$ & $6,378,137.0000 \mathrm{~m}$ \\
Semi-minor axis of the spheroid $(b)$ & $6,356,752.3142 \mathrm{~m}$ & $6,356,752.3141 \mathrm{~m}$ \\
Flattening of the spheroid $(f)$ & $1 / 298.257223563$ & $1 / 298.257222101$ \\
\hline
\end{tabular}

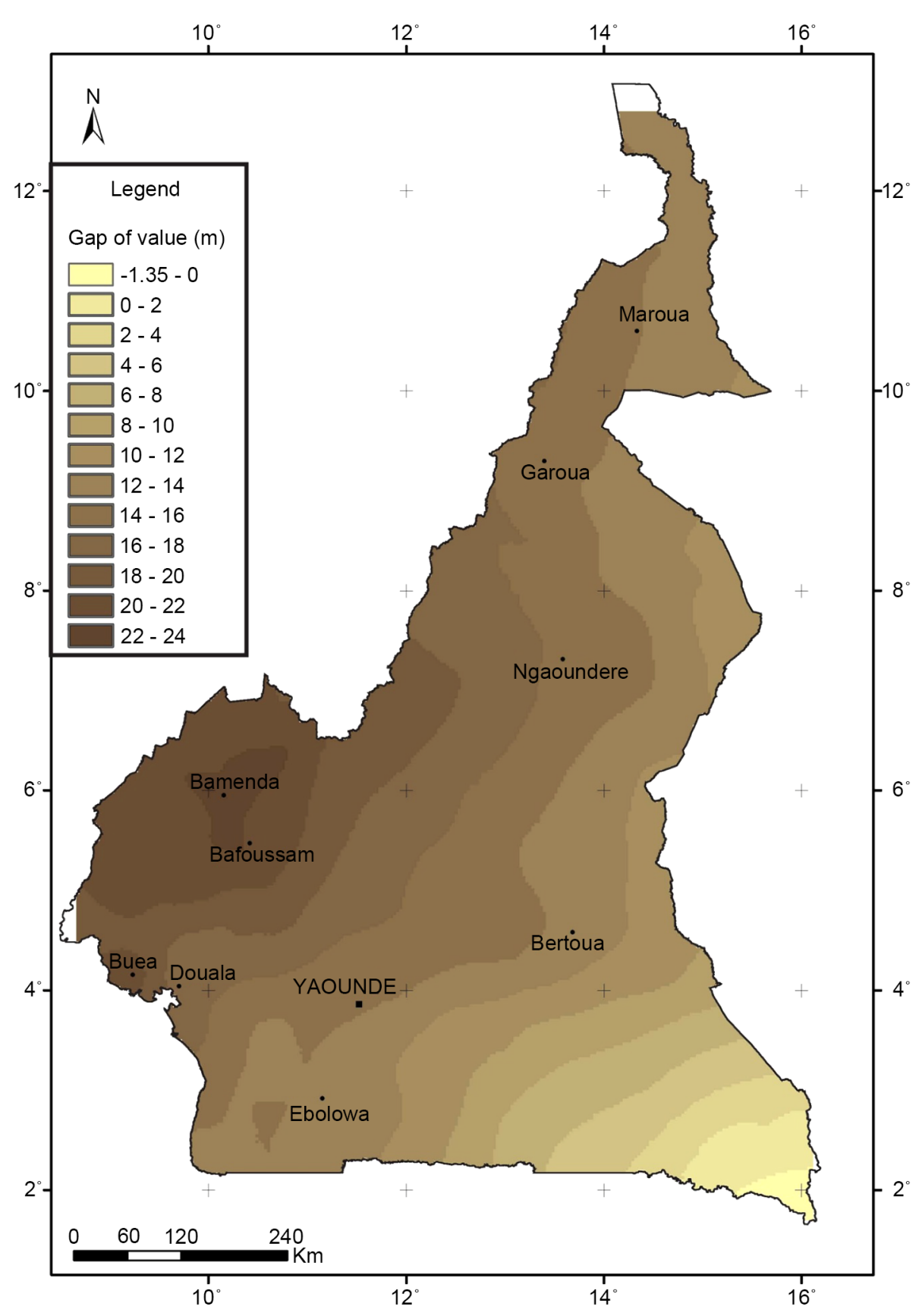

Figure 2. Residual map between the local ellipsoid model obtained from the global WGS84 ellipsoid where the parameters are well known and the geoid over Cameroon. 
Table 2. Parameters of the local ellipsoid obtained from the global WGS84 ellipsoid using the least square form of the Molodensky formula.

\begin{tabular}{cc}
\hline Parameters & Values \\
$\Delta X$ & 151.840979 \\
$\Delta Y$ & -88.1397904 \\
$\Delta Z$ & 215.810833 \\
$\Delta f$ & 0.0000959551031387562 \\
$\Delta a$ & 127.982768 \\
\hline
\end{tabular}

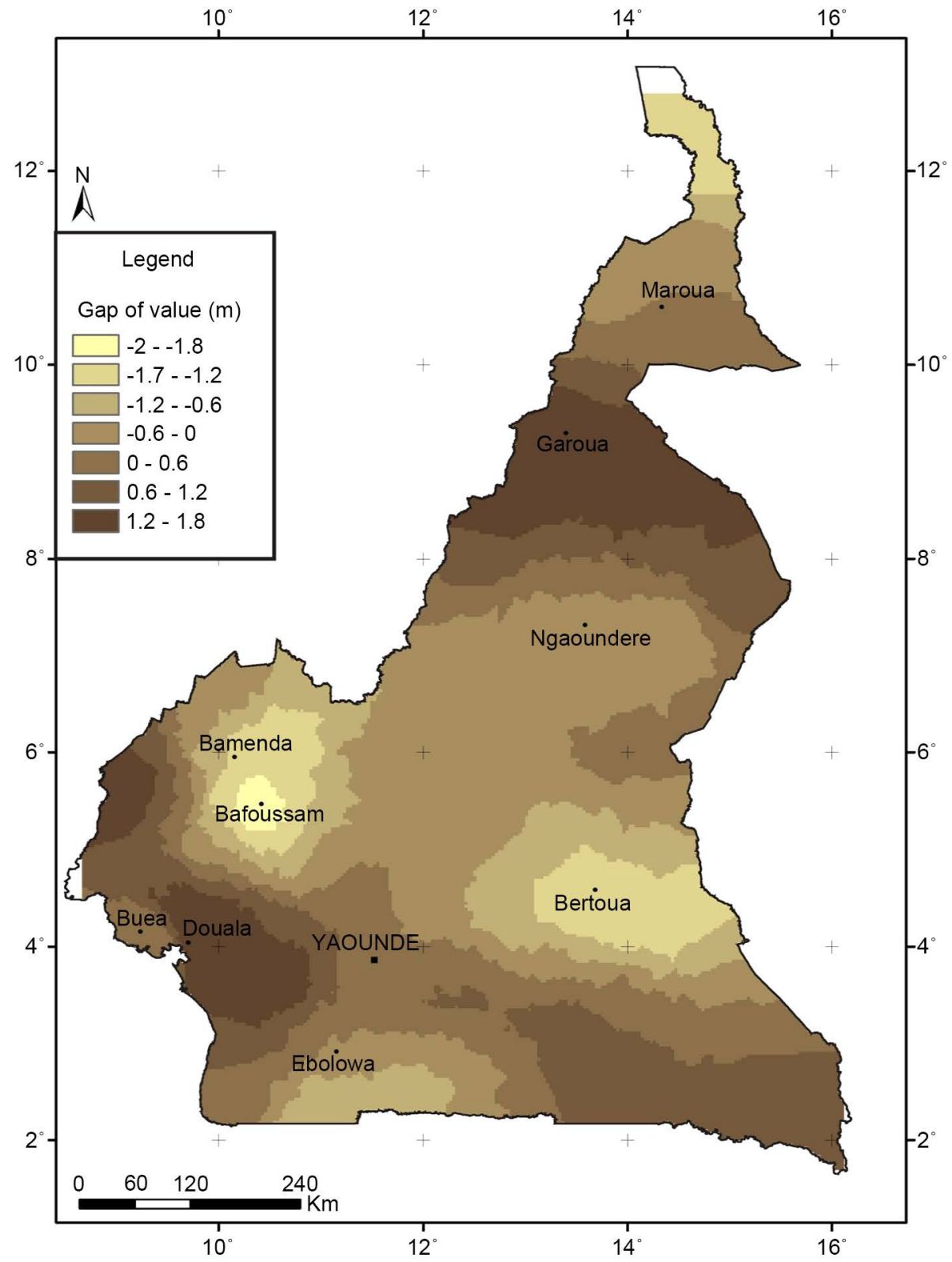

Figure 3. Residual map between the local ellipsoid model obtained from the global WGS84 ellipsoid and the geoid over Cameroon. The semi-major and semi-minor axis of the ellipsoid $\mathrm{a}$ and $\mathrm{b}$ respectively are assumed variables in order to ensure the best fitting of the geoid to the ellipsoid model. 
The geoid model (Cameroon Geoid Model 2011 (CGM11)) used in this scientific research [9], was adjusted from the global Earth Geoid Model 2008 (EGM08; [10]) and served as a reference surface in Cameroon. The CGM11 is more accurate than the local geoid model of Cameroon CGM05 computed by [3].

The difference between the local ellipsoid obtained from the global WGS84 ellipsoid and the geoid is shown in Figure 2.

It is important to note that the above map shows the gap values ranging from -1.35 to $24 \mathrm{~m}$ increase from south east to North West of the map. The gap values reach their peak over the west Cameroon Volcanic Line and centered at Bafoussam and Bamenda.

The processing of these data uses the Molodensky formula from which the least square method is applied as shown in the above paragraph. An equation in the form $A X=B$ is developed where $A$ is a $5 \times 5$ matrix with a number of points $N=555$. The resolution of this equation enables the determination of the local ellipsoid parameters for Cameroon from the parameters of the WGS84 ellipsoid with the standard deviation of $\sigma_{0}=1.072$. The results are summarized in the Table 2.

The above parameters aim to achieve the model residual map, which also corresponds to the difference between the ellipsoid model and the geoid shown in Figure 3. Note that the residual gap is small and comprises between $-2 \mathrm{~m}$ and $1.8 \mathrm{~m}$. The gap values are small in the area where the geodetic control points are densely distributed like in the localities of Bafoussam, Bamenda, Bertoua and Maroua.

The recent works conducted by [1], using the same data, have estimated the values of $\Delta X, \Delta Y, \Delta Z$, with a standard deviation of $1.679 \mathrm{~m}$. In that study, the semi-major and semi-minor axis $a$ and $b$ respectively, were assumed invariables and corresponded of the WGS84 ellipsoid. Therefore, $\Delta a=\Delta b=0$. In this case, $a$ and $b$ are assuming variables in order to ensure the best fitting of the geoid to the ellipsoid model. This allows to evaluate the values of $\Delta X, \Delta Y, \Delta Z, \Delta a, \Delta f$ with a standard deviation of $1.072 \mathrm{~m}$. These results improve significantly compare to those found by the previous author.

\section{Conclusion}

The parameters of the local reference ellipsoid for Cameroon were determined by the Molodensky analytical method using GPS data of the new geodetic network set in 2011. This method compares the parameters of two ellipsoids, and also determines the parameters of one ellipsoid from the known parameters of another using the least squares method. The results obtained show that the differences between the WGS84 ellipsoid parameters and the local ellipsoid are $\Delta x=151.840979, \Delta y=-88.1397904, \Delta z=215.810833, \Delta f=0.0000959551031387562$ and $\Delta a=127.982768$, assuming that the ellipsoid principal axis is pair-wise parallel and of the same orientation. These are determined with a standard deviation of $1.072 \mathrm{~m}$ between the two ellipsoids. These results improve significantly the similar study conducted by Foyang in 2010 which only determined the $\Delta x, \Delta y, \Delta z$ parameters, with a standard deviation of $1.679 \mathrm{~m}$, using the same method while assuming that $\Delta f=0$ and $\Delta a=0$.

\section{Acknowledgements}

The authors thank the Minister of State Property, Survey and Land Tenure, who has permitted them to use data set from Cameroon Geodetic Network.

\section{References}

[1] Foyang, L. (2012) Mise en place d'une Infrastructure cartographique de Base au Cameroun et son influence sur la cartographie et les mesures du parcellaire. Mémoire de Master professionnel, Université de Yaoundé I, Cameroun, 109 p.

[2] Yakubu, I. and Kumi-Boateng, B. (2015) Ramification of Datum and Ellipsoidal Parameters on Post Processed Differential Global Positioning System (DGPS) Data-A Case Study. Ghana Mining Journal, 15, 1-9.

[3] Kamguia, J., Tabod, C.T., Nouayou, R., Tadjou, J.M., Manguelle-Dicoum, E. and Kande, H.L. (2007) The Local Geoid Model of Cameroon, CGM05. Nordic Journal of Surveying and Real Estate Research, 4, 7-23.

[4] Dekin, R.E. (2004) The Standard and Abridged Molodensky Coordinate Transformation Formulae. Department of Mathematical and Geospatial Sciences, RMIT University, Australia.

[5] Molnár, G. and Timár, G. (2005) Determination of the Parameters of the Abridging Molodensky Formulae Providing the Best Horizontal Fit. Geophysical Research, 7, 01018. 
[6] Newsome, G.G. and Harvey, B.R. (2003) GPS Coordinate Transformation Parameters for Jamaica. Survey Review, 37, 218-234. http://dx.doi.org/10.1179/sre.2003.37.289.218

[7] Jean, H. (2009) Méthodes numériques et informatiques 2: Ajustements au sens des “moindres carrées”. Université Pierre et Marie CURIE, France.

[8] Molodensky, M.S. and Eremeev, V.F. (1960) A New Method of Solving Geodetic Problems. Trudy TsNIIGAiK.

[9] Carme, J.L. (2012) Le nouveau réseau géodésique national du Cameroun. Revue XYZ, Nº131, 2e trimestre 2012, 37-46.

[10] Pavlis, N., Holmes, S.A., Kenyon, S.C. and Factor, J.K. (2008) An Earth Gravitational Model to Degree 2160 : EGM08. Presented at the 2008 General Assembly of the European Geosciences Union, Vienna, April 2008, 13-18.

\section{Submit or recommend next manuscript to SCIRP and we will provide best service for you:}

Accepting pre-submission inquiries through Email, Facebook, LinkedIn, Twitter, etc.

A wide selection of journals (inclusive of 9 subjects, more than 200 journals)

Providing 24-hour high-quality service

User-friendly online submission system

Fair and swift peer-review system

Efficient typesetting and proofreading procedure

Display of the result of downloads and visits, as well as the number of cited articles

Maximum dissemination of your research work

Submit your manuscript at: http://papersubmission.scirp.org/ 